\title{
The Effects of Viewing an Uplifting 360-Degree Video on Emotional Well-Being Among Elderly Adults and College Students Under Immersive Virtual Reality and Smartphone Conditions
}

\author{
Qian Liu, PhD, ${ }^{1}$ Yanyun Wang, MS, ${ }^{2}$ Mike Z. Yao, PhD, ${ }^{2}$ Qingyang Tang, and Yuting Yang, BA ${ }^{1}$
}

\begin{abstract}
Positive emotions are important for the physical and mental health of elderly adults. Previous research has suggested that the emotion processing mechanisms of elderly adults differ from those of young people. The current study used a mixed-methods approach to explore the emotional impacts of viewing an uplifting $360^{\circ}$ videos under immersive (using a virtual reality [VR] headset) and nonimmersive (using a smartphone) conditions on elderly populations and young people. In Study 1, we conducted a pre-post treatment betweensubject field experiment (smartphone vs. VR) among 58 seniors (aged 60 years and above) in a community center in Beijing, China. One-on-one semistructured interviews of each participant were conducted after the experiment. In Study 2, we conducted a between-subject laboratory experiment with the same design among college students. The results show that while both VR and smartphone viewing conditions led to a significant positive effect on the self-reported emotions of the college students, only the smartphone viewing condition had a significant impact on the emotional well-being of the elderly participants. For college students, immersive VR had a stronger effect on inducing positive emotions than smartphone, but for elderly people, the smartphone viewing condition was more effective in improving emotional wellbeing than immersive VR. An analysis of the qualitative data from interviewing the elderly participants also revealed a divergence in the acceptance of VR technologies among elderly adults, and the sense of telepresence had both positive and negative effects on the elderly participants' emotions and experiences.
\end{abstract}

Keywords: virtual reality, mixed method, emotional well-being, elderly adults, college students

\section{Introduction}

$\mathbf{T}$ HE Well-BeING OF an aging population in a fastgrowing economy during a period of rapid modernization and technological innovation is a major social problem facing many societies today. Research has shown that the level of loneliness among elderlies is on the rise globally. ${ }^{1,2}$ Previous research has shown that smartphone use could improve human cognitive functions, ${ }^{3}$ and there were positive correlations between using smart digital products and elderly adults' overall cognitive function, visual-spatial executive function, attention, abstract thinking, delayed recall, and orientation ability. ${ }^{4}$

As an emerging means of communication, virtual reality (VR) technology has been used in health-related areas such as pain dispersion, ${ }^{5,6}$ psychological problems therapy, ${ }^{7-11}$ and health communication. ${ }^{12}$ For example, researchers have used VR-based exposure therapy to treat psychological disorders such as phobias, ${ }^{7,8}$ bulimia, ${ }^{9}$ depression, ${ }^{10}$ anxiety disorders, ${ }^{11}$ and have achieved positive results. In addition, researchers have used VR technology to simulate feelings similar to those experienced in panic disorder, depression, and disability to enhance empathy among the general population toward those with the conditions. ${ }^{13}$ Some researchers have also applied new technologies to elderly adults hoping to improve their living standards. For example, game-based VR training programs designed to help patients with impaired cognitive functions perform daily routines led to significant improvements among older people unfamiliar with such technologies. ${ }^{14}$ In a comparison between traditional fitness

\footnotetext{
${ }^{1}$ School of Journalism and Communication, Beijing Normal University, Beijing, People's Republic of China.

${ }^{2}$ Institute of Communications Research, College of Media, University of Illinois, Urbana-Champaign, Illinois.
} 
training and a 3D augmented reality training system (3DARS) in the rehabilitation of lower limb function and balance problems among elderly people, the 3D-ARS group showed a stronger healing effect. ${ }^{15}$

Previous studies have shown promising results in the applications of VR among elderly people, but they mostly focused on using VR as a tool in medical treatments. As VR technologies and devices are becoming widely accessible, there is a need to study VR as a source of entertainment and information in everyday use. While there has been ample research showing that VR could provide a stronger sense of telepresence ${ }^{16,17}$ and affected emotions, ${ }^{18}$ most of the studies were conducted among student participants, and we did not know whether these effects would be found in other age groups.

Past researchers have found that negative and positive virtual environments could stimulate corresponding negative and positive emotions, respectively, among college students; negative virtual environments were also more likely to cause corresponding emotional changes than were positive virtual environments. ${ }^{18}$ As people age, their recognition, attention, and memory of negative emotions such as anger, sadness, and fear would decline, but the positive emotions are less affected. ${ }^{19}$ Differences in the aging effect of emotion processing could also be found in other research areas. For example, researchers found that older people were more strongly influenced than younger people by message framing in risk-related decision-making ${ }^{20}$ and such age-related difference in decision-making could be attributable to a difference in the processing of emotional information. ${ }^{19}$

Our study adds to the literature by focusing on the positive effects of VR as a source of entertainment on the emotional well-being among the elderly population. Can VR give elderly adults positive experiences, lift their emotions, and promote physical and mental health as it would for younger people? Motivated by this general question, the current study aims to address two specific research questions: (a) How would different $360^{\circ}$ video-viewing devices (VR vs. smartphone) affect the emotions of elderly people? and (b) How would such effects differ between elderly adult and college student groups?

\section{Study 1}

Method

In Study 1, we conducted a pre-post treatment (viewing an uplifting $360^{\circ}$ video) between-subjects (devices: iPhone 8 vs. Pico 4K G2 VR goggles) field experiment among elderly people aged 60 years or above. For the smartphone viewing condition, we used the app UtoVR, which is a smartphonebased $360^{\circ}$ video player. The field experiment was conducted in a middle-class senior community with over 7,000 residents. Considering elderly adults' health conditions and safety, we performed the experiment in the community activity center rather than inviting the participants to our laboratory. Each participant received a 50-RMB (about $\$ 7$ in USD) cash coupon as a reward after finishing the study.

\section{Participants}

The participants were recruited with the help of the community service center. The center sent our research no- tice to all the residents. Sixty-two participants signed up and participated in the study. One participant dropped out of the study, and three were disqualified because they were younger than 60 years of age. Ultimately, 58 valid cases were included in the subsequent data analysis. Among all the valid cases, the youngest was 60 years old, and the oldest was 91 years old $\left(M_{\mathrm{age}}=68.84, S D=7.095\right)$. Among them, 36 were female and 22 were male. Table 1 provides additional demographic information.

\section{Procedure}

The study was conducted in a dedicated room in the community activities center. A group of trained research assistants assisted with the data collection. Upon their arrival to the experiment room, all participants were asked to read and sign the consent form. Where the participant had difficulty reading or understanding the consent form, the experimenters read the information out loud and provided additional verbal explanation. Next, the participants were asked to complete a pretest survey containing questions about their mood and emotions. Then, participants were randomly assigned to view an uplifting $360^{\circ}$ video on a smartphone or an immersive VR headset. The video, called $V R$ China was $\sim 8$ minutes long. It was produced by National Geographic (China) and contained sceneries from major natural and cultural attractions in China. After watching the short video, the participants completed a posttest survey asking about their mood, emotions, and sense of presence. Upon completion of the experiment, the experimenter conducted a 30 minutes semistructured interview with each participant about their feelings and viewing experience. At the conclusion of the study, the participants were thanked and debriefed. The participants in the smartphone condition were invited to see the video in VR if they were interested.

\section{Results}

\section{Quantitative results}

Previous studies have found that male and female differ in the ways they experience VR and in the levels of perceived presence. $^{21,22}$ To check for a potential gender effect, a chisquare test was conducted to check whether male and female participants were randomly assigned to the VR and smartphone. There was no significant difference in gender distribution between the two conditions $\left[\chi^{2}(1, N=58)=2.78\right.$, $p=0.096]$. We then focused on the two research questions in the subsequent analyses.

Emotions. Using the Positive and Negative Affect Schedule (PANAS) ${ }^{23}$ scale, positive and negative emotion scores were calculated separately for each participant using 20 items (10 positive and 10 negative). The positive and negative emotion scores were computed by summing the scores for the 10 items (with each item on a 1-5 scale). To test whether the VR and smartphone viewing conditions affected the elderly participants' positive and negative emotions, a mixed analysis of variance (ANOVA) was conducted using positive and negative emotion scores as the dependent variables, time (pre-post viewing) as the within-subject 
Table 1. Demographics of Study 1 and Study 2

\begin{tabular}{lccccccr}
\hline & Male & Female & Total & Average age & Minimum age & Maximum age & Study \\
\hline Elderly people & $22(38 \%)$ & $36(62 \%)$ & 58 & 68.84 & 60 & 91 & 1 \\
Undergraduates & $30(50 \%)$ & $30(50 \%)$ & 60 & 20.4 & 18 & 25 & 2 \\
\hline
\end{tabular}

factor and group (VR vs. smartphone) as the between-subject factor.

First, the results showed a significant effect of pre-post viewing on emotional change. There was a significant increase in positive emotion scores after the participants viewed the video $[F(1,56)=12.12, p=0.001]$. To explore whether this increase in positive emotions was significant for both the VR group and the smartphone group, we split the data by group, and two repeated measures analyses were conducted separately. We found that only the elderly participants in the smartphone viewing condition showed a significant increase in positive emotions $\left[M_{\text {pre }}=29.29\right.$, $S D=8.03 ; \quad M_{\text {post }}=34.46, \quad S D=8.69 ; \quad F(1,29)=18.20$, $p=0.000]$. However, there was no significant change in positive emotions among participants in the VR group $\left[M_{\text {pre }}=30.1, \quad S D=8.00 ; \quad M_{\text {post }}=30.03, S D=8.72 ; \quad F(1\right.$, 29) $=1.43, p=2.41]$ (Fig. 1).

Next, we examined the between-group and pre-post differences in the changes in negative emotion scores. Similarly, the results showed an overall significant effect of pre-post viewing on reported negative emotions. Negative emotion scores decreased after the elderly participants watched the video via smartphone $[F(1,56)=12.62$, $p=0.001]$. However, we only found this significant prepost viewing decrease in negative emotion scores in the smartphone group $\left[M_{\text {pre }}=14.11, S D=4.18 ; M_{\text {post }}=11.96\right.$, $S D=3.39 ; F(1,29)=18.52, p=0.000]$. For the elderly participants in the VR group, the decrease in negative emotion $\left(M_{\text {pre }}=14.5, S D=4.80 ; M_{\text {post }}=12.87, S D=4.97\right)$ was not statistically significant $[F(1,29)=3.18, p>0.05]$ (Fig. 2).

In summary, results from the quantitative analyses show that viewing an uplifting $360^{\circ}$ video increased positive emotion and decreased negative emotion, but only in the smartphone viewing condition. The elderly participants in the VR viewing condition did not experience any significant change in their emotions after viewing the same video.

\section{Qualitative analyses}

To further explore factors that might have affected elderly adults' emotions and experiences, we conducted one-on-one semistructured interviews with each participant. The interview questions cover different topics, such as the sense of presence, technology use experience, feelings/memories about and attitudes toward the stimulus video, and digital media use habits. We conducted interviews in standard Mandarin and transcribed $\sim 30$ hours of interview recordings. For privacy, all the personal information from the interviews was removed and a pseudonym was assigned to each participant. We conducted open coding, axial coding, and selective coding step by step suggested by Corbin and Strauss. ${ }^{24}$

Positive and negative effects caused by VR experiences. An analysis of the interviews showed that the elderly participants in the VR group split on how they felt the immersive viewing experience. About half of them reported a positive viewing experience. Three themes of divergence emerged from those who reported discussed VR positively: (a) a strong feeling of being there (presence), (b) the ease of using the equipment, and (c) the wide visual field offered by VR.

About half of the respondents disliked using VR and thought it was not suitable for elderly adults. Some respondents reported that the strong sense of presence made them feel scared and anxious. Some reported physical discomfort, such as dizziness, and that wearing the machine was uncomfortable. The vision problems of elderly adults and the $4 \mathrm{~K}$ clarity of the head display itself also affected the experience. In addition, quite a few elderly participants said that they preferred watching TV. They showed resistance to new technologies and thought that watching TV was easier and safer. Overall, six themes emerged from these negative experiences of VR viewing: (a) low perceived presence caused by distraction, (b) physical discomfort, (c) blurred vision, (d) a preference for other digital media, (e) the sense of surrealness, and (f) the feelings of strangeness and resistance to new technology.

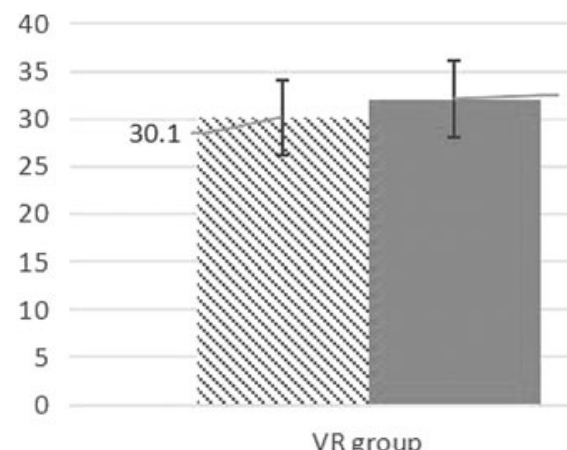

VR group

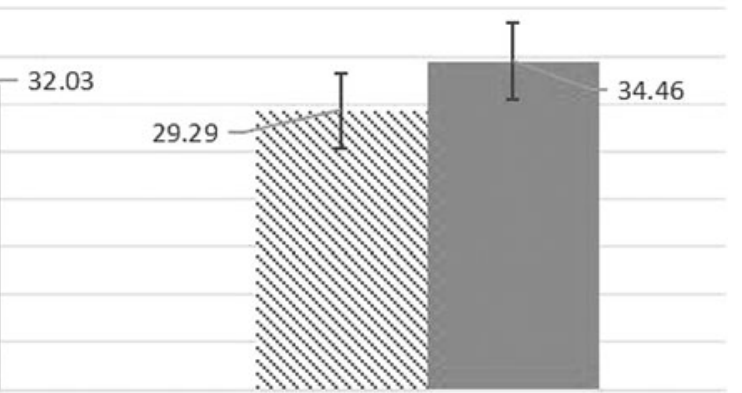

Smartphone group
FIG. 1. Means of positive emotion scores of the elderly between devices (VR vs. smartphone) and across time (pretest vs. posttest). VR, virtual reality. 
FIG. 2. Means of negative emotion scores of the elderly between devices (VR vs. smartphone) and across time (pretest vs. posttest).

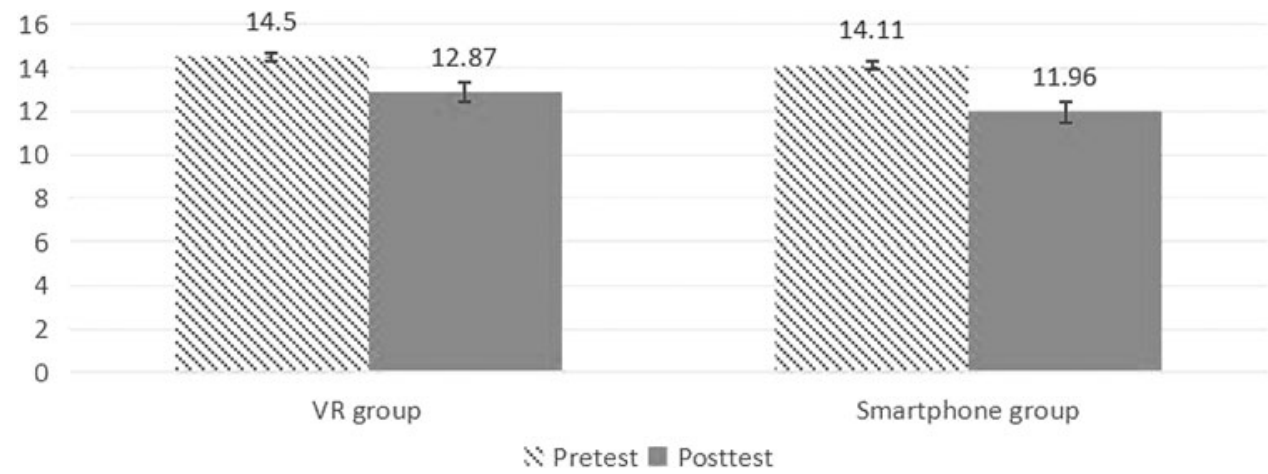

While the uplifting stimulus video increased positive emotions and decreased negative emotions under the smartphone viewing condition, which had offered lower presence, lower graphic quality, and a much narrower viewing field than immersive VR, this effect was not found in the VR viewing condition. The findings from our qualitative analysis of participants' responses during the postexperiment interview provided insights into the mixed results from the quantitative analyses. Table 2 summarizes the themes emerged from participants in both VR and smartphone conditions.

\section{Study 2}

Study 2 aimed to further explore how VR technologies might impact the elderly users and young people differently. A pre-post between-subject laboratory experiment with the same design, quantitative measures, and same stimulus video and equipment as in Study 1 was conducted among college students.

\section{Method}

With the assistance from university staff, a recruitment notice was posted in several WeChat groups at a major public university in China. We targeted student groups maintained by the academic departments and student interest groups. The participants signed up for the study through a link embedded in the recruitment notice. Sixty student participants completed the study within 1 week from April 15 to 21,2019 . Their ages ranged from 18 to 25 years $\left(M_{\text {age }}=\right.$ $20.38, S D=2.164)$. Thirty male and 30 female participants were randomly distributed in the conditions $\left[\chi^{2}(1,60)=2.40\right.$, $p>0.5]$.

\section{Results}

\section{Emotions}

The same analytic methods used for the elderly sample were applied to the student data. Self-reported positive and negative emotion scores were calculated separately for each participant. A mixed-model ANOVA was performed using the average positive emotion score and the average negative emotion score as the dependent variables, time (pretest vs. posttest) as a within-subject variable and viewing condition (VR vs. smartphone) as a betweensubject variable.
Pre-post viewing had a significant main effect on both the positive emotions and negative emotions. Participants reported an increase in the positive emotions $[F(1,58)=21.14$, $p=0.000]$ and a decrease in the negative emotions $[F(1$, $58)=48.02, p=0.000]$ after viewing the uplifting promo video regardless of the viewing condition.

Repeated measures analyses were conducted to determine whether there was a difference in the emotional impacts of the video between the VR group and the smartphone group. In contrast to the elderly participants' in Study 1, the positive emotional impact was found in both viewing conditions. For the students in the VR group, the average positive emotion score increased from 30.2 $(S D=7.14)$ to $34(S D=6.55)[F(1,29)=21.93, p=0.000]$, and their negative emotion score decreased from 17.93 $(S D=7.90) \quad$ to $13.80 \quad(S D=5.15) \quad[F(1,29)=22.96$, $p=0.000]$. For those in the smartphone group, their positive emotion scores increased from $29.27(S D=5.22)$ to 31.53 $(S D=7.49)[F(1,29)=4.746, p=0.038]$, and their negative emotion score decreased from $18.73(S D=8.16)$ to 14.10 $(S D=5.01)[F(1,29)=25.07, p=0.000]$.

\section{Emotions and age}

To get a better understanding that how devices (VR vs. smartphone) influence emotions differently in the two age groups (college students vs. elderly adults), we combined the two data sets and conducted a series of two-way analysis of covariance (ANCOVA) analyses. The first ANCOVA was conducted with postpositive emotion score as the dependent variable, age group (college students vs. elderly adults) and device (VR vs. smartphone) as independent variables, and pretest positive emotion as a covariate. The result revealed a significant interaction effect $[F(1,113)=4.458, p=0.037]$. More specifically, college students in VR group reported higher positive emotions $(M=34, S D=6.55)$ than those in the in smartphone group $(M=31.54, S D=7.487)$, while elderly adults in smartphone group reported higher positive emotion scores $(M=34.46, S D=8.68)$ than those in the in VR group $(M=32.03, S D=8.72)$, after controlling the preexisted emotions. The second ANCOVA was conducted with the same setting but negative emotion score as the dependent variable. However, there is no significant result associated with it. In conclusion, VR video has a better effect on rising positive emotions than smartphone video for young generation, but for elderly people, smartphone video has a better effect than VR experience (Fig. 3). 


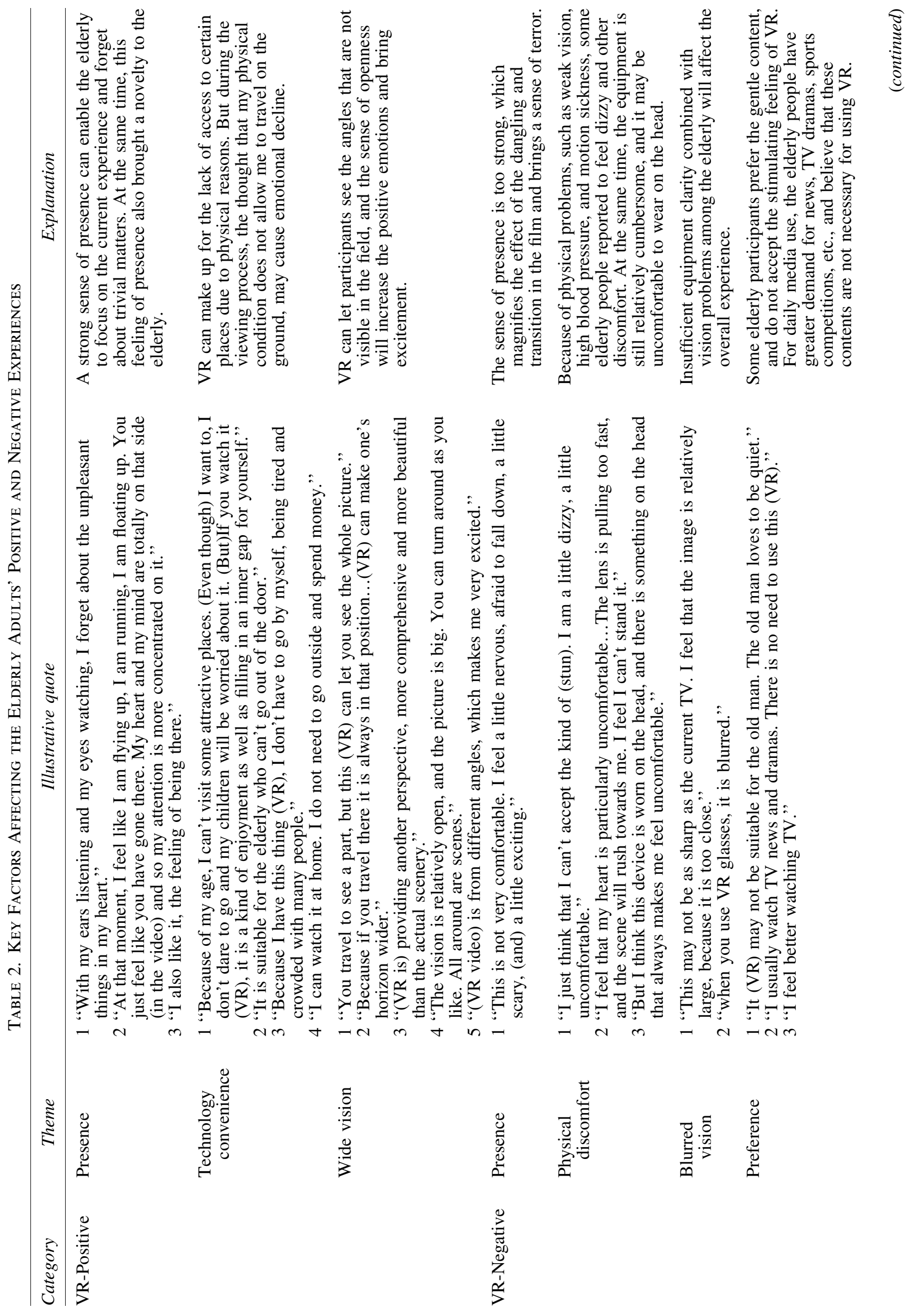




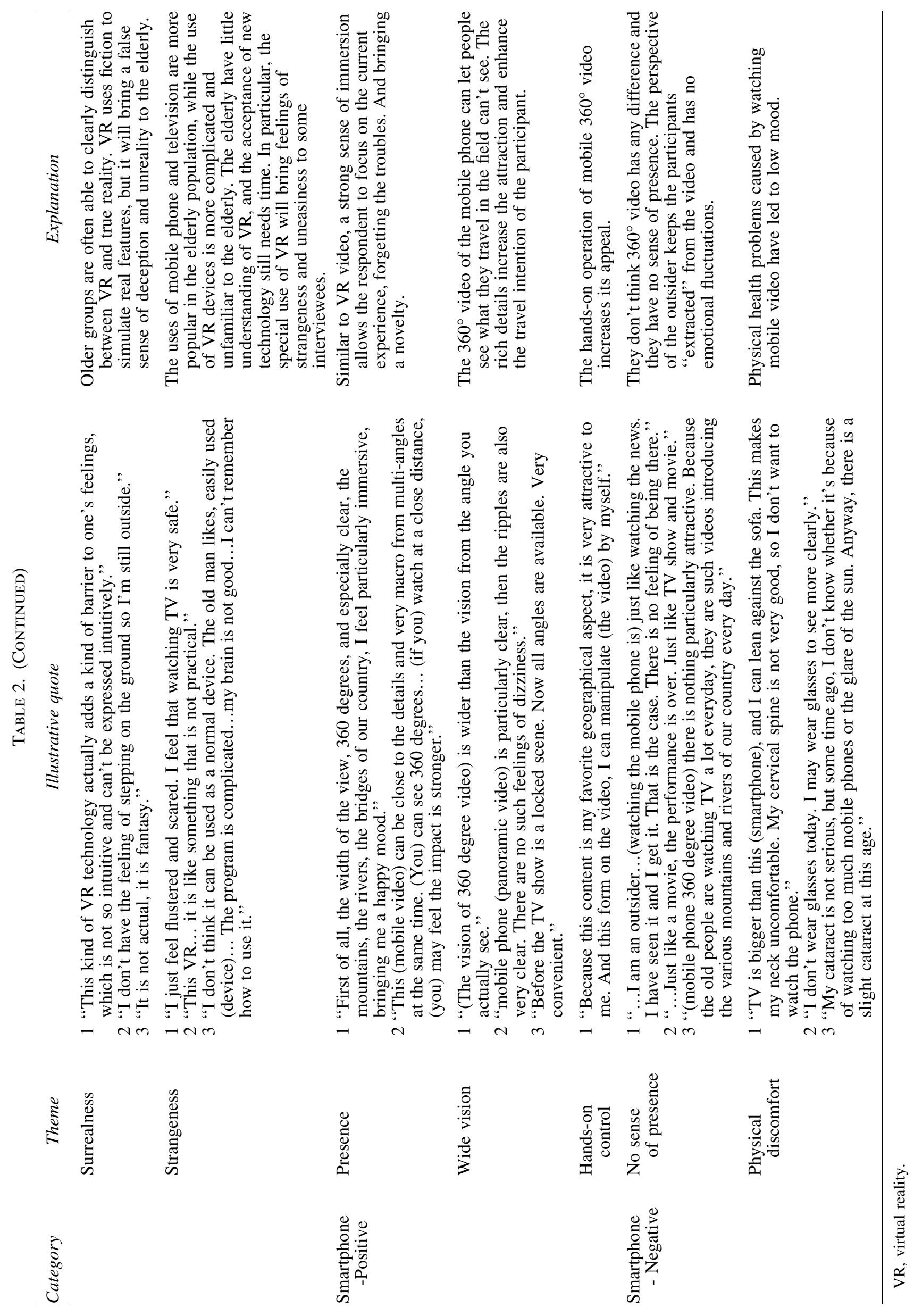




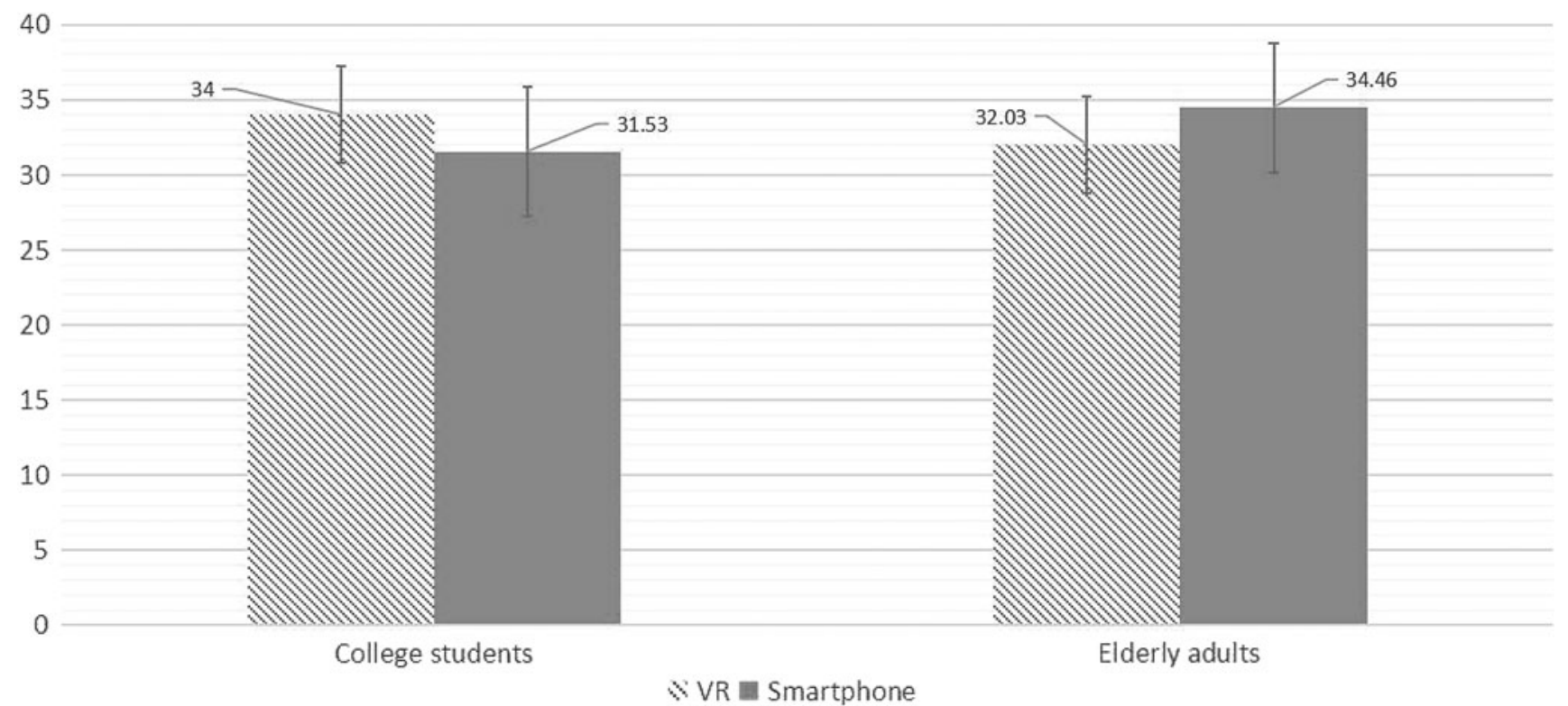

FIG. 3. ANCOVA for positive emotions among age (college students vs. elderly adults) groups and device (VR vs. smartphone) groups. ANCOVA, analysis of covariance.

\section{Discussion}

Our research has both theoretical and practical implications. First, this study revealed that while an enhanced sense of presence from viewing an uplifting $360^{\circ}$ video in the immersive environment of a VR positively affected young people's emotional well-being, such a positive effect among the elderly adults is limited. For the young generation, immersive VR was more effective than the mobile smartphone in inducing positive emotions, which is consistent with findings from previous studies. ${ }^{16,17}$ However, for the elderly, viewing the same video on the smartphone brought more positive emotions than immersive VR. These findings bring to light the nuances specific to an understudied population in a seemingly robust effect found other populations. At the current rate of adoption, VR may soon be as popular as TV and smartphones today in people's everyday lives. Our research provides a practical reference for future developments of the VR technology for prosocial applications. Findings from our study suggest that it is necessary to consider the needs of special populations, such as the elderly, in future development and applications of the VR technology.

Besides the impacts from the viewing devices, another explanation for the age-related effect of emotion processing could be the strategy change hypothesis. ${ }^{19}$ The elderly participants could have been proactively using a different emotional processing strategy that was used by the younger participants based on previous experiences. ${ }^{25,26}$ Our findings supported the strategy change mechanism to some extent. In contrast to young people who like to try new things, the qualitative analysis of our interviews with the elderly adults showed that older people seemed to prefer familiar and safer activities, such as watching TV, for mood management. The novelty and exciting of new media technology may not have had a strong impact on positive emotions among the elderly participants as they did among the younger population.

There were several limitations of this study. First, we collected the experimental data of study 1 in a community center instead of in a laboratory. The open environment of the community center may have affected the results. Gender effect has been ruled out in our study design, but it can be an important factor to be explored in future study. Second, the sample size in both studies was fairly small, and randomization might not have been entirely successful. This problem could be addressed in future replications of our study by including additional and similar videos in the stimuli material. Finally, our comparison of the young and elderly samples was not statistically robust due to differences in experimental setting, methodological control, and data collection procedures.

\section{Acknowledgments}

We thank the No. 43 Youse Community Service Centre for their help and National Geography China for providing the video for study.

\section{Author Disclosure Statement}

No competing financial interests exist.

\section{Funding Information}

The study was supported by "the Fundamental Research Funds for the Central Universities"' (Grant No. 310422106), and "Beijing Social Science Fund" (Grant No. 19XCC015).

\section{References}

1. Wang M, Zhou Y, Wang L, et al. Advances in mental health interventions for empty nesters. Journal of Nursing 2015; 30:107-110.

2. Grenade L, Boldy D. Social isolation and loneliness among older people: issues and future challenges in community and residential settings. Aust Health Rev 2008; 32:468-478.

3. Wilmer HH, Sherman LE, Chein JM. Smartphones and cognition: a review of research The links between mobile 
technology habits and cognitive functioning. Front Psychol 2017; 8:605.

4. Lu S, Lin Wei, Li H, et al. Research on the relationship between the use of hobbies and smart products and their cognitive functions in elderly care institutions. Chinese Journal of Practical Nursing 2018; 34:407-411.

5. Mosso Vázquez JL, Mosso Lara D, Mosso Lara JL, et al. Pain distraction during ambulatory surgery: virtual reality and mobile devices. Cyberpsychology, Behavior, and Social Networking 2019; 22:15-21.

6. Hu B, Huang J, Li R. The effect of virtual reality on the psychological state of patients undergoing gastroscopy. Chinese Endoscopy 2018; 24:23-27.

7. Suso-Ribera C, Fernández-Álvarez J, García-Palacios et al. Virtual reality, augmented reality, and in vivo exposure therapy: a preliminary comparison of treatment efficacy in small animal phobia. Cyberpsychology, Behavior, and Social Networking 2019; 22:31-38.

8. Tardif N, Therrien C, Bouchard S. Re-examining psychological mechanisms underlying virtual reality-based exposure for spider phobia. Cyberpsychology, Behavior, and Social Networking 2019; 22:39-45.

9. Ferrer-Garcia M, Pla-Sanjuanelo J, Dakanalis A, et al. A randomized trial of virtual reality-based cue exposure second-level therapy and cognitive behavior second-level therapy for bulimia nervosa and binge-eating disorder: outcome at six-month follow-up. Cyberpsychology, Behavior, and Social Networking 2019; 22:60-68.

10. Liu C, Liu W, Liu T, et al. Application of virtual reality in the treatment of anxiety and autism. Journal of System Simulation 2015; 27:2233-2238.

11. Felnhofer A, Hlavacs H, Beutl L, et al. Physical presence, social presence, and anxiety in participants with social anxiety disorder during virtual cue exposure. Cyberpsychology, Behavior, and Social Networking 2019; 22:46-50.

12. Jung S, Lee J, Biocca F, et al. Augmented reality in the health domain: projecting spatial augmented reality visualizations on a perceiver's body for health communication effects. Cyberpsychology, Behavior, and Social Networking 2019; 22:142-150.

13. Wiederhold BK. Is augmented reality the next frontier in behavioral health? Cyberpsychology, Behavior, and Social Networking 2019; 22:101-102.

14. Gamito P, Oliveira J, Morais D, et al. Cognitive stimulation of elderly individuals with instrumental virtual realitybased activities of daily life: pre-post treatment study. Cyberpsychology, Behavior, and Social Networking 2019; 22:69-75.

15. Ku J, Kim YJ, Cho S, et al. Three-dimensional augmented reality system for balance and mobility rehabilitation in the elderly: a randomized controlled trial. Cyberpsychology, Behavior, and Social Networking 2019; 22:132-141.
16. Sas C, Gregory M. Presence equation: an investigation into cognitive factors underlying presence. Presence: Teleoperators and Virtual Environments 2003; 12:523-537.

17. Slater M, Sylvia W. A framework for immersive virtual environments (FIVE): speculations on the role of presence in virtual environments. Presence: Teleoperators and Virtual Environments 1997; 6:603-616.

18. Yang Y, Zhang Z, Lin L. The impact of positive and negative virtual environments on college students' emotions. Chinese Journal of Sports Medicine 2013; 32:708714.

19. Li H, Ding N, Dong Q. The neural mechanism of aging effects in emotional processing. Advances in Psychological Science 2009; 17:356-361.

20. Kim S, Goldstein D, Hasher L, et al. Framing effects in younger and older adults. The Journals of Gerontology 2005, 60B:215-218.

21. Lombard M, Reich RD, Grabe ME, et al. Presence and television: the role of screen size. Human Communication Research 2000; 26:75-98.

22. Felnhofer A, Kothgassner OD, Beutl L, et al. Is virtual reality made for men only? Exploring gender differences in the sense of presence. Proceedings of the International Society on Presence Research, Philadelphia, PA, 2012; 103-112.

23. Watson D, Clark LA, Tellegen A. Development and validation of brief measures of positive and negative affect: the PANAS scales. Journal of Personality and Social Psychology 1988; 54:1063-1070.

24. Corbin JM, Strauss A. Grounded theory research: procedures, canons, and evaluative criteria. Qualitative Sociology $1990 ; 19: 418-427$.

25. Mather M, Canli T, English T, et al. Amygdala responses to emotionally valenced stimuli in older and younger adults. Psychological Science 2004; 15:259-263.

26. Fischer H, Sandblom J, Gavazzeni J, et al. Age-differential patterns of brain activation during perception of angry faces. Neuroscience Letters 2005; 386:99-104.

Address correspondence to: Dr. Qian Liu School of Journalism and Communication Beijing Normal University Room 9307, Jingshi Hotel 19 Xinjiekouwai Street Haidian District Beijing 100875 People's Republic of China

E-mail: qianliu@bnu.edu.cn 